\title{
EXPRESSION OF ADRENOCEPTOR SUBTYPES IN THE PRETERM PIG HEART
}

\author{
M.Y. Kim ${ }^{1}$, Y.A. Eiby ${ }^{1}$, E.R. Lumbers ${ }^{1,2}$, A.C. Boyce ${ }^{2}$, K.J. Gibson ${ }^{2}$, B.E. Lingwood ${ }^{3}$
}

${ }^{1}$ The University of Queensland, Brisbane, QLD, ${ }^{2}$ University of New South Wales, Sydney, NSW, ${ }^{3}$ University of Queensland Centre for Clinical Research, The University of Queensland, Herston, QLD, Australia

Background: Sympathetic effects on the fetal heart appear to be predominantly mediated through $\alpha$ adrenoceptors ( $\alpha$-ARs) whereas in adults $\beta$-adrenoceptors ( $\beta$-ARs) dominate. If $\beta$-adrenoceptors are absent or scarce in the preterm heart this may limit the ability of the preterm heart to respond to inotropic treatments.

Aim: To compare mRNA expression of AR subtypes in preterm and term neonatal piglets.

Methods: Piglets were delivered by Caesarean section at 92 days (preterm group) and 114 days of gestation (term group, full term $\approx 115$ days). mRNA levels of AR subtypes, normalised to GAPDH, were measured in left and right ventricle by real-time PCR using Taqman ${ }^{\odot}$ chemistry (Applied Biosystems). Levels were expressed relative to a calibrator using the comparative $\mathrm{C}_{\mathrm{T}}$ method.

Results: In the left ventricle, expression of $\beta_{1}$-ARs was lower in preterm than in term hearts $(P<0.001)$ but $\alpha_{1 D^{-}}$and $\alpha_{2 A^{-}}$AR expression was higher compared to term hearts $(P<0.001)$ (Fig 1- mean \pm SEM). $\alpha_{1 A^{-}}$AR expression was not different between preterm and term hearts.
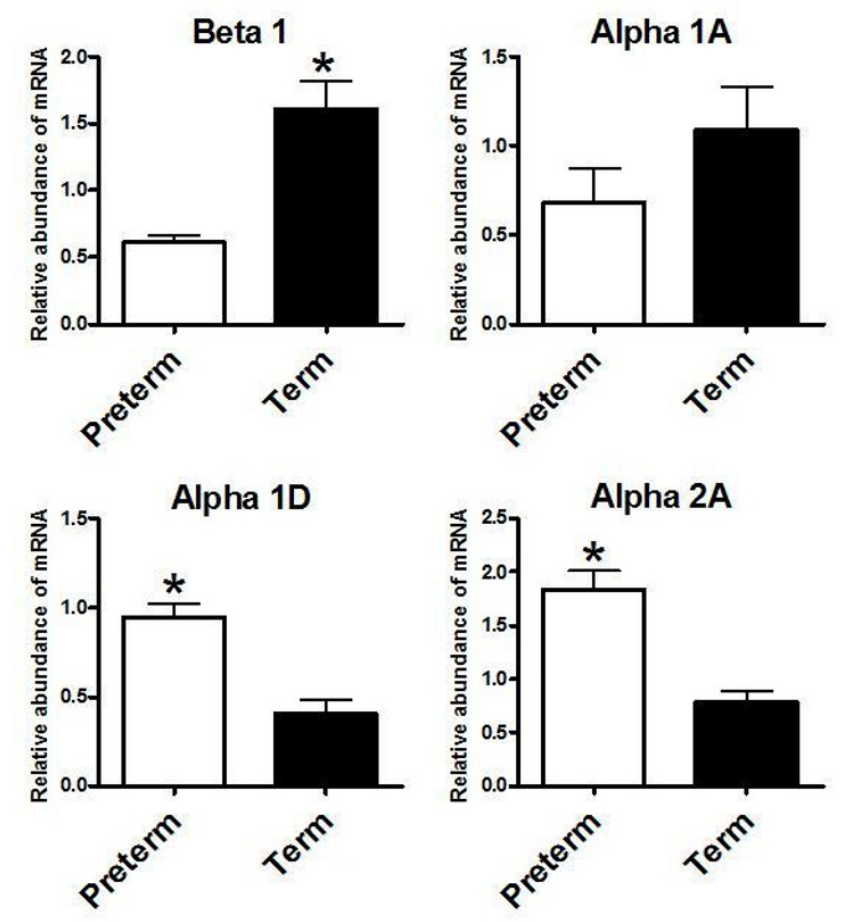

\section{[Figure 1]}

Expression in right ventricle was similar to left (data not shown). $\alpha_{2 \mathrm{~B}}$-ARs were absent in all groups.

Conclusions: The lower expression of $\beta_{1}$-AR in preterm pig hearts compared to term may explain the inefficient cardiac function seen after premature birth and also why some preterm babies do not respond to conventional inotrope treatments that act on $\beta_{1}$ adrenoceptors. 